\title{
Functional Assessment of Hearing Aid Benefit: Incorporating Verification and Aided Speech Recognition Testing into Routine Practice
}

\author{
Sarah A. Sydlowski, Au.D., Ph.D., M.B.A., ${ }^{1}$ Michelle King, ${ }^{1,2}$ \\ Karen Petter, Au.D., ${ }^{1}$ and Meagan Lewis Bachmann, Au.D. ${ }^{3}$
}

\section{ABSTRACT}

Potential cochlear implant (CI) candidates arrive to the clinic with a variety of hearing loss configurations, hearing aid history, and aided capabilities. CI candidacy is primarily determined based on aided speech recognition capability, which relies on benefit derived from use of hearing aids. Therefore, contemporary evaluation for CI candidacy should incorporate a battery of testing to determine abilities and limitations and must be predicated on appropriate verification of the hearing aid fitting. However, recent reports, including a retrospective chart review of patients presenting to Cleveland Clinic for CI evaluation, suggest that a significant subset of patients may be using inappropriately fit or programmed amplification. Thus, a combination of simulated real-ear measurements and aided speech recognition testing is essential for fully assessing the effect of amplification and ultimately determination of CI candidacy. Furthermore, waiting to incorporate these tools until CI candidacy is suspected may delay timely identification of problems or need to change technology. Utilization of evidence-based decision drivers ultimately leads clinicians to timely patient-specific interventions which may include surgical intervention or other amplification options. As audiology moves into a healthcare era in which payers consider the benefit of our services to overall health and well-being, demonstrating timely, optimal outcomes using thorough, multifactorial evaluation is essential.

${ }^{1}$ Cleveland Clinic - Head and Neck Institute, Cleveland, Ohio; ${ }^{2}$ Case Western Reserve University, Cleveland, Ohio; ${ }^{3}$ Wake Forest Baptist Medical Center, Winston-Salem, North Carolina.

Address for correspondence: Sarah Sydlowski, Au.D., Ph.D., MBA, Cleveland Clinic, Head and Neck Institute, 9500 Euclid Avenue, Desk A71, Cleveland, OH 44195 (email: sydlows@ccf.org).
Barriers to Cochlear Implant Access: Acknowledging the Challenges, Changing the Future; Guest Editor, Sarah Sydlowski, Au.D., Ph.D., M.B.A.

Semin Hear 2021;42:365-372. (C) 2021. Thieme. All rights reserved. Thieme Medical Publishers, Inc., 333 Seventh Avenue, 18th Floor, New York, NY 10001, USA DOI: https://doi.org/10.1055/s-0041-1739369.

ISSN 0734-0451. 
KEYWORDS: hearing aid verification, cochlear implant candidacy, aided speech recognition

Although cochlear implantation $(\mathrm{CI})$ is a well-accepted and long-standing option for the management of individuals with sensorineural hearing loss who receive limited benefit from hearing aids, the complex challenge of underutilization of the technology ${ }^{1,2}$ and inconsistent determination of candidacy exists. ${ }^{3}$ Although there have been some attempts to identify correlation between unaided audiometric audibility and speech recognition and eventual CI candidacy, ${ }^{4-7} \mathrm{CI}$ candidacy is primarily determined based on aided speech recognition capability, which relies on benefit derived from use of hearing aids. While early candidacy criteria suggested appropriate candidates should demonstrate no measurable benefit from hearing aids, candidacy criteria have expanded to include individuals who demonstrate limited benefit from hearing aids. ${ }^{8,9}$ However, the definition of limited benefit is not universally consistent. The Minimum Speech Test Battery (MSTB), developed in 1996 and later updated in 2011, recommends assessment of the ability to understand monosyllabic words, sentences in quiet, sentences in a fixed level of noise, and sentences in pseudo-adaptive levels of noise. Functionality is also evaluated in multiple conditions (right-aided, left-aided, and bilaterally aided). ${ }^{10-12}$ This comprehensive battery is important in understanding the capabilities of each ear, binaural benefits that may be realized, the effect of context on speech understanding, the effectiveness of hearing aids, and the consideration of assistive listening devices or implantable devices. Regardless of which metrics are used to ultimately define benefit delivered via amplification as compared with the potential for increased benefit delivered via CI, essential to a reliable analysis is the core understanding that the hearing aid(s) used for the determination of candidacy are providing adequate audibility to result in the best possible performance in the given listening condition. Because aided testing determines candidacy for an irreversible surgical procedure that has the potential to decrease unaided natural hearing, it is imperative that assessment of hearing aid benefit is conducted using optimally fit hearing aids.

\section{HEARING AID VERIFICATION}

Hearing aid verification has long been touted as the gold standard for confirmation of appropriate hearing aid fitting. On-ear verification takes into account both the output of the instrument and the physical characteristics of the ear canal to assure that the signal that is delivered provides sufficient audibility, measured by approximation of targets (e.g., NAL-NL1 or DSL) and the associated Speech Intelligence Index (SII). Simulated real-ear measures offer similar estimations but rely on average real ear to coupler differences. ${ }^{13}$ Over 20 years ago, Newall and Oliver ${ }^{14}$ concluded that "the consequence of fitting hearing aids without following a systematic procedure is usually poor aided performance. Indeed, poor aided performance may be the result of inappropriate amplification and may lead to the erroneous conclusion that hearing aids are of little to no benefit and that a cochlear implant is therefore needed or may negate the outcome of the cochlear implant evaluation.” Although most individuals being considered for CI candidacy have experience using hearing aids, Holder et $\mathrm{al}^{15}$ reported that 177 of 287 patients in their clinic over a 2-year timeframe arrived without hearing aids, citing lack of perceived benefit. The perceived lack of benefit could be attributed to inconsistency in appropriateness of fit for those potential candidates using hearing aids, which has also been reported. Prentiss et al collected survey responses from audiologists conducting CI evaluations who reported that when hearing aids were verified, they met prescriptive targets less than half the time. ${ }^{16,17}$ Holder et $\mathrm{al}^{15}$ reported that only 32 of 110 (29.1\%) of patients' own hearing aids met NAL-NL2 target audibility for $60 \mathrm{~dB}$ SPL speech in their own clinic during CI evaluations. These results are perhaps not surprising, given that in a survey of 533 hearing aid users only $36 \%$ participated in verification and validation measures during their hearing aid 
fitting appointments despite the fact that an estimated $75 \%$ of audiologists purchase real-ear verification equipment. ${ }^{16}$

Mueller and $\mathrm{Hall}^{13}$ estimated that approximately $70 \%$ of patients are fit with hearing devices based on a manufacturer's estimated first fit rather than settings that have been objectively verified, although it is generally acknowledged that first-fit hearing aid settings vary drastically from one manufacturer to the other. Gain has been shown to differ by as much as $20 \mathrm{~dB}$ depending on the manufacturer ${ }^{18}$ and can significantly change speech audibility at individual frequencies. ${ }^{19}$ In fact, Valente et $\mathrm{al}^{20}$ noted that when subjects compared a first-fit hearing aid program to one verified using probe microphone measures, 79\% of subjects preferred that program. Not surprisingly, subjects also showed significant improvements in phoneme and word recognition compared with the first-fit approach.

CI centers may be a unique niche within audiology where the essential nature of verification is fully appreciated and utilized by more than $90 \%$ of audiologists prior to candidacy evaluation. ${ }^{16}$ Both the American Academy of Audiology Cochlear Implant Practice Guidelines ${ }^{21}$ and the Guidelines for Best Practice in the Audiological Management of Adults with Severe and Profound Hearing Loss ${ }^{22}$ recommend probe microphone measures or simulated realear verification. When hearing aids do not meet prescriptive targets, $94 \%$ of respondents in a recent survey of CI audiologists purport to follow these standards by programing and verifying clinic loaner aids to use for testing. ${ }^{15}$ It is often not possible to reprogram the patient's existing hearing aid for candidacy testing, because they do not currently use one, because they are using a personal sound amplification device or a proprietary hearing aid that can only be adjusted by certain providers, or because earmold tubing is hardened and requires excessive modification. For these reasons, some centers opt to preemptively program and verify clinic hearing aids and conduct aided testing in multiple conditions (i.e., with patient hearing aid(s) and with verified clinic hearing aid(s)) to estimate the optimization of the hearing aid fitting. This latter model is the protocol used by audiologists in the Cleveland Clinic Hearing Implant Program. Aided CNC Word testing ${ }^{23}$ is conducted in both the right-aided and leftaided conditions with both the patient's own hearing aid(s) and clinic hearing aids that have been programmed and verified to meet NALNL1 or NAL-NL2 targets using simulated real-ear measures. In a recent retrospective review of 179 patients (321 ears, $48 \%$ female, $52 \%$ male, ages $18-94$, mean $=68.89$ years) seen for CI evaluation, clinically significant improvement on CNC Word scores was determined by using a SPRINT chart modeling binomial distribution of speech scores for a 25 -word list. ${ }^{24}$ On a group level, a paired $t$ test did not suggest statistically significant differences between scores using patient hearing aids versus clinic hearing aids. However, on an individual level, clinically significant differences were noted for 99 of the 321 ears analyzed when using clinic versus patient hearing aids (Fig. 1). For 85 ears, patients understood words better with clinic hearing aids and 14 ears had poorer understanding. The remaining 220 ears demonstrated clinically insignificant changes with scores falling within the $95 \%$ confidence interval for patient aid compared with clinic aid. For those ears in which clinically significant improvement was noted, improvement ranged from 12 to $88 \%$ (mean $=33.85 \%)$. In multiple cases, word recognition improved from $<10 \%$ to $>90 \%$ which, from a counseling perspective, was very impactful from the patient perspective. Importantly, had a comparison between hearing aids not been undertaken, and only patient hearing aids used for the CI evaluation, at least 28 ears may have been recommended CI when in fact, word understanding scores $>50 \%$ could be obtained with appropriately fit hearing aids.

As previously noted, some clinics utilize simulated or real ear verification to confirm that hearing aids are meeting prescriptive targets and do not test with multiple sets of hearing aids. It would generally be assumed that if hearing aids are meeting targets, then best outcomes can be expected. In the current review, 221 ears had real-ear measures completed on their current and clinic programmed hearing aids. Of 221 patient aids, 178 were not meeting target (defined as being within $5 \mathrm{~dB}$ ). For those patients with significant differences in word recognition when using different hearing aids, $67 \%$ were not meeting targets, while $12 \%$ were 


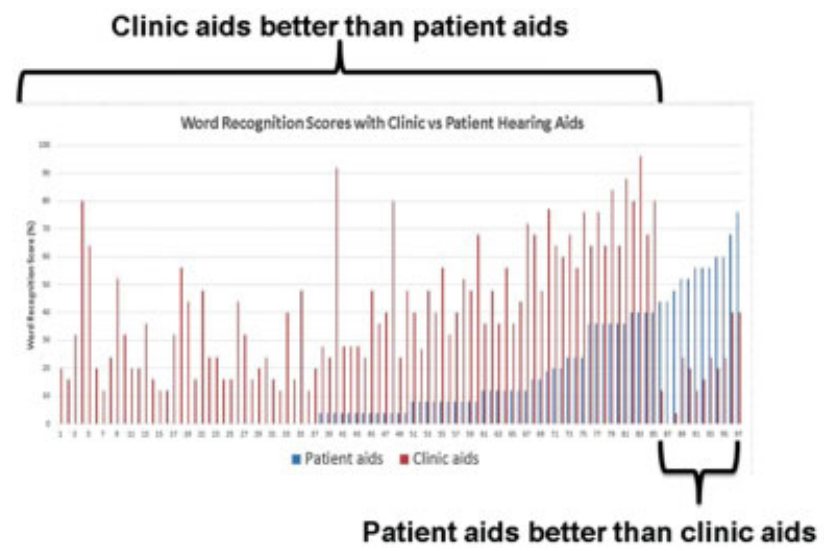

Figure 1 Word recognition scores with clinic versus patient hearing aid (HA).

meeting targets, and the rest were not specifically evaluated. Surprisingly, for those who did not demonstrate significant differences, 51\% were not meeting targets and $14 \%$ were meeting targets, which is fairly similar to the group who did have significant differences. This finding suggests that meeting targets alone may not be sufficient to expect best outcomes. Interestingly, six ears performed clinically significantly better with their own hearing aids that were not meeting targets compared with clinic programmed and verified hearing aids that did meet target. This result makes sense because real-ear measurements only confirm that the device output is adequate to ensure audibility, but aided speech recognition testing involves higher level processing. Similarly, if only realear verification was completed, a subset of patients still would not have demonstrated best aided performance with their own hearing aids.

As evidenced earlier, only by using a battery of measurements can we maximize patient performance, whether with hearing aid technology or to recommend implantable devices. Patients seek audiologic intervention because they want to improve their hearing, whether it is in the form of a CI or hearing aid. Using all of the tools at our disposal determines which intervention will be most effective. It is evident that for a subset of patients seen for CI evaluation, optimizing device settings and maximizing audibility results in improved speech understanding; however, it is essential to un- derstand not only the effect of optimizing audibility, but also assessing the role of higher-level processing on performance. It was only through the combination of simulated real-ear measures for hearing aid verification and aided speech recognition testing that best possible aided performance was quantified and a determination relative to $\mathrm{CI}$ candidacy could be made.

\section{LIMITATIONS OF RELYING ON UNAIDED TESTING TO IDENTIFY CI CANDIDATES}

A significant challenge for clinicians is knowing when to send potential candidates for implant evaluation. Historically, clinicians tend to consider $\mathrm{CI}$ as a last resort, only referring when they feel confident that the patient is receiving no benefit from hearing aids and will be a certain candidate. This approach leads to under-referral and under-utilization of CI. Ideally, there would be a more objective measure that is commonly assessed in a comprehensive diagnostic battery that would trigger a clinician to suspect CI. Several studies have undertaken to identify such a surrogate measure, with varying results. McRackan et $\mathrm{al}^{4}$ prospectively sought to describe a correlation between word recognition conducted unaided using NU-6 words under earphones and aided NU-6 word ${ }^{25}$ scores obtained in the soundfield. Of 94 study participants, word recognition scores for $50 \%$ of participants $(N=47)$ were higher in the earphone condition 
than in the aided condition, leading the authors to conclude that the common clinical practice of using word recognition scores measured with earphones to predict aided speech recognition scores or hearing aid benefit cannot be supported. McRackan et $\mathrm{al}^{5}$ took this analysis one step further by retrospectively considering the relationship of word recognition conducted unaided using CNC words under earphones and aided $\mathrm{CNC}$ word scores obtained in the sound field as part of a CI evaluation. They found that earphone to aided differences ranged from -38 to $72 \%$ (mean $14.3 \pm 19.9 \mathrm{~dB}$ ) with $51.9 \%$ demonstrating earphone scores that were significantly higher than aided word recognition scores, which might have disqualified potential candidates from pursuing CI evaluation. Of those whose unaided scores were $>50 \%$, $82.6 \%$ were CI candidates despite seemingly adequate unaided word recognition. Once again, the authors concluded that unaided word recognition testing has limited diagnostic value for CI candidacy and that earlier and routine measurement of aided word recognition may help guide clinical decision making by determining the extent to which patients are achieving maximum benefit with their hearing aids or should consider CI. More recently, Zwolan et $\mathrm{al}^{6}$ sought to evaluate the effectiveness of using a screening guideline of a pure tone average (PTA) $\geq 60 \mathrm{~dB}$ in the better ear and $\leq 60 \%$ unaided word recognition to identify traditional CI candidates. The authors concluded that more than $90 \%$ of the patients who qualified for a CI demonstrated a PTA in the better ear that is greater than or equal to $60 \mathrm{~dB}$ HL. Additionally, of the candidates for whom a better ear unaided monosyllabic word score was available, 92\% demonstrated a score less than or equal to $60 \%$. However, the authors concede a very key point which is that "it should be noted that these guidelines do not apply to patients who may receive a CI outside of traditional indications, such as those with asymmetric hearing loss (AHL), single-sided deafness (SSD), or those who receive a recommendation for a CI off-label." Thus, it is incumbent upon clinicians to realize that relying on this measure will only assist them in identifying traditional candidates who meet more stringent candidacy guidelines. With the well-recognized limita- tions of the existing unaided diagnostic battery for determination of potential CI candidacy, it may be time to reimagine the standard battery from a functional perspective.

\section{CONSIDERATIONS FOR BEST \\ PRACTICE: FUNCTIONAL HEARING ASSESSMENT VERSUS CI EVALUATION}

As the overall goal of communication assessment is ultimately to improve the individual's functionality, functional testing should be approached from the standpoint of efforts to maximize the current communication abilities, rather than simply a binary choice of implant or no implant. Although historically the clinical model has been to introduce aided speech recognition testing as part of a CI evaluation, incorporating aided speech recognition testing as a standard component of hearing aid fitting and maintenance offers value in several ways (e.g., reinforcing provider trust, accurately determining candidacy for hearing aid and CI technology, and optimizing healthcare cost and time efficiencies).

In terms of reinforcing provider trust, an oft-repeated anecdote in CI clinics is that of the patient who presents for CI evaluation, frustrated because they have invested in multiple sets of hearing aids, each costing thousands of dollars. While their hearing loss is upsetting, often what rises to the surface in these patient stories is the distrust in their hearing aid provider that has emerged, despite months or years of best effort on the part of the clinician to "dig to the bottom of the toolkit" and try every amplification option. Anecdotally, it is not uncommon to hear "I wish I'd known about CI sooner" as opposed to "I wish I'd tried hearing aids longer." Incorporating aided speech recognition testing as part of the hearing aid fitting and maintenance process confirms to the patient that their concerns (difficulty hearing in noise, differences in understanding with male vs. female talkers) are being heard and evaluated. In combination with real-ear measures, providers have an opportunity to demonstrate the value of the services audiologists can offer by confirming audibility, assessing speech understanding in multiple environments, and 
making recommendations to adjust accordingly. Furthermore, having longitudinal speech recognition results available enables a savvy clinician to refer for $\mathrm{CI}$ as soon as a patient might be eligible, not as a last resort when all other interventions have failed, because changes in performance are readily apparent. Not only does the thoroughness of this evaluation make an impression on patients and encourage their ongoing trust in their provider and recognition of hearing loss management as more than a device, aided speech recognition testing also introduces cost-savings to the hearing healthcare system. Although aided speech recognition testing can be time-consuming ( $\geq 30$ minutes/ patient), the associated information, if appropriately interpreted and addressed, can save months of hearing aid adjustments, expenditures on new, less than optimal technology, and patient frustration, all of which patients and payers would prefer to avoid.

\section{VALUE-BASED CARE AND AUDIOLOGY SERVICES}

As healthcare continues to evolve in our country, one increasingly compelling proposition is that of "value-based care." ${ }^{26}$ Some larger corporations have designated centers throughout the country where their employees can receive care for higher priced procedures. They have chosen these particular institutions after thorough vetting and critical examination of outcomes. In essence, they are paying for a greater likelihood of a positive result as evidenced by data and patient satisfaction. Similarly, accountable care organizations (ACO) are groups of physicians, hospitals, and other healthcare providers who come together voluntarily to provide coordinated high-quality care to their Medicare patients. The goal of coordinated care is to ensure that patients get the right care at the right time, while avoiding unnecessary duplication of services and preventing medical errors. When an ACO succeeds both in delivering high-quality care and spending health care dollars more wisely, the ACO will share in the savings it achieves for the Medicare program. ${ }^{27}$ As healthcare evolves and these costsaving sharing models become more predominant, audiologists should be prepared to dem- onstrate the value evidence-based practice creates for patients and payers. To most, value would be defined as better hearing and reaching their hearing goals, ideally as efficiently and cost-effectively as possible. Certainly this is the case for those seeking a CI evaluation. The addition of measures such as functional testing, probe microphone measures, and subjective assessments leads the clinician's management decisions including but not limited to recommending a CI, new hearing aid technology or reprogramming of existing hearing aids, endorsing remote microphone or FM technology for improvement with speech in noise, and counseling regarding communication strategies. Additionally, aided speech recognition testing, more than any other component of the standard audiologic battery, confirms for the patient that the audiologist is hearing and assessing their real-life concerns and ability to succeed in a variety of listening environments.

A limiting factor in the universal adoption of aided speech recognition testing to optimize outcomes and identification of CI candidates is that while some audiologic services are covered by commercial insurances, others, including functional testing for the purpose of fitting a hearing aid, may not be because there is a not a specific code available to describe this procedure. Since January 2020, the CPT code 92626, evaluation of auditory rehabilitation status, has been redefined to refer specifically to aided speech recognition testing conducted for the purpose of identifying candidacy for or monitoring progress with an implantable hearing device. However, such functional information is valuable in that it demonstrates the patient's ability to benefit with hearing technology and considers higher level process in addition to device output. Although a specific code for this purpose does not currently exist, the fact that accurate coding options are lagging behind clinical practice should not deter providers from incorporating sound clinical judgment and utilization of appropriate measures. Aided testing can be billed just as many noncovered hearing aids and services are currently billed to the patient using an unlisted code or potentially on a cash-pay basis until coding catches up with best practices. Particularly given that there is poor correlation of unaided pure tone and 
speech recognition measures for the identification of any but traditional CI candidates, avoidance of functional aided testing is likely to contribute to the underutilization of CI technology.

\section{CONCLUSION}

Optimizing patient outcomes, delivering costeffective, efficient care, and building patientprovider trust are best accomplished by incorporating evidence-based clinical standards in audiologic practice. In the case of CI candidacy, it is essential to confirm that hearing aid(s) used for the determination of candidacy are providing adequate audibility to result in the best possible performance in a given listening condition. Many patients present for a CI evaluation using hearing aids that are not meeting target audibility or are not delivering optimal speech recognition testing. Additionally, providers may find it difficult to identify potential CI candidates (particularly those with substantial residual hearing, AHL, SSD, or other off-label indications) using unaided audiometric measures due to the poor correlation of CI candidacy with traditional diagnostic measures. To avoid this dilemma and introduce earlier, more consistent identification of opportunities to offer better amplification or CI, aided speech recognition testing should be incorporated as part of the standard hearing aid management protocol.

\section{CONFLICT OF INTEREST}

S.A.S. is a consultant for Oticon Medical and Cochlear.

\section{REFERENCES}

1. Simpson AN, Matthews LJ, Cassarly C, Dubno JR. Time from hearing aid candidacy to hearing aid adoption: a longitudinal cohort study. Ear Hear 2019;40(03):468-476

2. Sorkin DL. Cochlear implantation in the world's largest medical device market: utilization and awareness of cochlear implants in the United States. Cochlear Implants Int 2013;14 Suppl 1 (Suppl 1):S4-12

3. Deep NL, Dowling EM, Jethanamest D, Carlson ML. Cochlear implantation: an overview. J Neurol Surg B Skull Base 2019;80(02):169-177
4. McRackan TR, Ahlstrom JB, Clinkscales WB, Meyer TA, Dubno JR. Clinical implications of word recognition differences in earphone and aided conditions. Otol Neurotol 2016;37(10):1475-1481

5. McRackan TR, Fabie JE, Burton JA, Munawar S, Holcomb MA, Dubno JR. Earphone and aided word recognition differences in cochlear implant candidates. Otol Neurotol 2018;39(07):e543-e549

6. Zwolan TA, Schvartz-Leyzac KC, Pleasant T. Development of a 60/60 guideline for referring adults for a traditional cochlear implant candidacy evaluation. Otol Neurotol 2020;41(07):895-900

7. Gubbels SP, Gartrell BC, Ploch JL, Hanson KD. Can routine office-based audiometry predict cochlear implant evaluation results? Laryngoscope 2017;127(01):216-222

8. Arnoldner C, Lin VYW. Expanded selection criteria in adult cochlear implantation. Cochlear Implants Int 2013;14(Suppl 4):S10-S13

9. Gifford RH, Dorman MF, Shallop JK, Sydlowski SA. Evidence for the expansion of adult cochlear implant candidacy. Ear Hear 2010;31(02):186-194

10. Advanced Bionics LLC, Cochlear Americas, MED-EL Corporation. Minimum speech test battery for adult cochlear implant users, 2011. Available at: http://www.auditorypotential.com/ MSTBfiles/MSTBManual2011-06-20\%20.pdf. Published June 2011

11. Luxford WMAd Hoc Subcommittee of the Committee on Hearing and Equilibrium of the American Academy of Otolaryngology-Head and Neck Surgery. Minimum speech test battery for postlingually deafened adult cochlear implant patients. Otolaryngol Head Neck Surg 2001;124(02): 125-126

12. Spahr AJ, Dorman MF, Litvak LM, et al. Development and validation of the AzBio sentence lists. Ear Hear 2012;33(01):112-117

13. Mueller HG, Hall JW. Audiologists' Desk Reference. San Diego: Singular Publishing Group; 1998

14. Newall P, Oliver J. Hearing aid optimization in the evaluation of cochlear implant candidacy. Adv Otorhinolaryngol 2000;57:281-283

15. Holder JT, Reynolds SM, Sunderhaus LW, Gifford RH. Current profile of adults presenting for preoperative cochlear implant evaluation. Trends Hear 2018;22:2331216518755288

16. surname>Kochkin S. MarkeTrak VIII: Reducing patient visits through verification \& validation. The Hearing Review Available at: https://www. hearingreview.com/hearing-products/marketrak-viiireducing-patient-visits-through-verification-ampvalidation. Published February 2, 2018. Accessed August 11, 2021

17. Prentiss S, Snapp H, Zwolan T. Audiology practices in the preoperative evaluation and management of adult cochlear implant candidates. JAMA Otolaryngol Head Neck Surg 2020;146(02):136-142 
18. Mueller HG, Picou EM. Survey examines popularity of real-ear probe-microphone measures. Hear J 2010;63:27-32

19. Hawkins DB, Cook JA. Hearing aid software predictive gain values: how accurate are they? Hear J 2003;56:26-34

20. Valente M, Oeding K, Brockmeyer A, Smith S, Kallogjeri D. Differences in word and phoneme recognition in quiet, sentence recognition in noise, and subjective outcomes between manufacturer first-fit and hearing aids programmed to NALNL2 using real-ear measures. J Am Acad Audiol 2018;29(08):706-721

21. Messersmith JJ, Entwisle L, Warren S, Scott M. Clinical practice guidelines: cochlear implants. J Am Acad Audiol 2019;30(10):827-844

22. Turton L, Souza P, Thibodeau L, et al. Guidelines for best practice in the audiological management of adults with severe and profound hearing loss. Semin Hear 2020;41(03): 141-246

23. Peterson GE, Lehiste I. Revised CNC lists for auditory tests. J Speech Hear Disord 1962; 27:62-70

24. Thornton AR, Raffin MJ. Speech-discrimination scores modeled as a binomial variable. J Speech Hear Res 1978;21(03):507-518

25. Tillman TW, Carhart R. An expanded test for speech discrimination utilizing CNC monosyllabic words. Northwestern University Auditory Test No. 6. Tech Re SAM-TR. 1966:1-12

26. Porter ME. What is value in health care? $\mathrm{N}$ Engl J Med 2010;363(26):2477-2481

27. Accountable Care Organizations (ACOS). CMS. Accessed August 11, 2021 at: https:/www.cms.gov/ Medicare/Medicare-Fee-for-Service-Payment/ACO 